\title{
Implantable Cardiac Devices and the Acute Care Management of Decompensated Heart Failure
}

\author{
Brian Hiestand · Said Laribi · Alexandre Mebazaa
}

Published online: 26 February 2013

(C) Springer Science+Business Media New York 2013

\begin{abstract}
The use of implantable cardiac devices in patients with chronic heart failure is on the rise. These devices perform a number of functions including pacing, defibrillation, and data collection. While referral for consideration of such devices can occur on an outpatient basis, an encounter in the acute care setting may be the one opportunity for getting an appropriate patient to the right device therapy. Thus, we will briefly discuss the indications for the various implantable cardiac devices. We will also discuss the data that can be extracted from implantable devices and describe how this information can assist with the diagnosis and treatment of acutely decompensated heart failure.
\end{abstract}

Keywords Acutely decompensated heart failure . Emergency department - Observation unit - Defibrillator . Pacemaker · Cardiac resynchronization therapy

B. Hiestand $(\square)$

Department of Emergency Medicine, Wake Forest University Health Sciences, 2120 Meads Hall Medical Center Boulevard, Winston-Salem, NC 27157, USA

e-mail: bhiestan@wfubmc.edu

\section{S. Laribi}

Emergency Department, APHP, Hôpital Lariboisière and Inserm U942, 2 Rue Ambroise Paré, 75010 Paris, France

e-mail: said.laribi@1rb.aphp.fr

\section{A. Mebazaa}

Department of Anesthesiology and Critical Care, APHP, Hôpital Lariboisière and Inserm U942, 2 Rue Ambroise Paré, 75010 Paris, France

e-mail: alexandre.mebazaa@1rb.aphp.fr

\section{Introduction}

Over the past 20 years heart failure hospitalizations have increased more than $150 \%$, with more than $75 \%$ of the patients admitted through the emergency department (ED) $[1 \bullet \cdot 2]$. Accurate and rapid diagnosis and therapy improves outcomes in decompensated heart failure [3]. However, dyspnea is hardly specific to decompensated heart failure. It is often a substantial challenge for the acute care physician to quickly determine whether the patient presenting with shortness of breath is experiencing decompensated heart failure or one of a multitude of other conditions that may present with dyspnea. Many heart failure patients managed in the acute setting have implantable cardiac devices. Others may meet criteria for having an implantable device but have not been considered for implantation. Data from implantable cardiac devices, if present, may assist in this endeavor. In this paper, we will briefly discuss the indications for the various implantable cardiac devices, as referral for consideration of such devices can be done on a non-emergent outpatient basis. In the second part we will discuss in more detail the data that can be extracted from implantable devices and describe how this information can assist with the diagnosis and treatment of acutely decompensated heart failure. The encounter in the acute care setting may be the one opportunity for getting an appropriate patient to the appropriate device treatment modality. Better knowledge of implantable devices can help the acute care physician to better manage patients with an acutely decompensated heart failure (ADHF) episode who have such devices.

\section{Therapeutic Functions}

The active functions of implantable devices can be broadly divided into two categories-arrhythmia termination and 
primary pacing. Defibrillation is the primary mode for termination of malignant ventricular tachydysrhythmias, although overdrive pacing may be attempted based on the capabilities of the device. Patients with heart failure are at risk for both atrial and ventricular tachydysrhythmias. The annual incidence of sudden cardiac death (SCD) in the USA is estimated at 2 per $1,000(0.2 \%)$ [4]; in patients with inducible dysrhythmias and chronic heart failure due to ischemia (the highest risk subgroup), that incidence climbs to more than $30 \%$. Other high-risk groups include cardiac arrest survivors and those with a left ventricular ejection fraction (LVEF) less than $35 \%$ and heart failure [5]. In the latter group, SCD comprises about $50 \%$ of all deaths [6].

Patients with heart failure who have survived ventricular tachycardia (VT), ventricular fibrillation (VF), or SCD are at high risk for recurrence. Regardless of the degree of underlying structural disease (preserved vs. decreased systolic function) or etiology (ischemic vs. non-ischemic cardiomyopathy), implantable cardioverter-defibrillator (ICD) implantation is recommended when quality of life and prognosis are such that SCD prevention is a desirable goal [7]. It should be noted that secondary prevention is not indicated in all patients-those with end-stage heart failure will not benefit from ICD implantation as death is likely, regardless of dysrhythmia protection.

Primary prevention refers to the use of an ICD in a patient who is deemed to be at substantial risk but has not yet sustained VT/VF or cardiac arrest. Multiple trials have demonstrated the superiority of ICD over medical therapy for primary prevention of SCD in the heart failure population. The Multicenter Automatic Defibrillation Trial (MADIT I) [8] demonstrated a substantial survival benefit in patients with heart failure (LVEF $\leq 35 \%$ and New York Heart Association [NYHA] classes I-III), previous myocardial infarction, and inducible VT, as compared to nonstandardized medical therapy (hazard ratio [HR] 0.46, $95 \%$ confidence interval [CI] 0.26-0.82).

MADIT II also focused on ischemic cardiomyopathy (LVEF $\leq 30 \%$ and prior myocardial infarction), but removed dysrhythmia as an inclusion criteria [9]. MADIT II demonstrated a survival benefit in terms of all-cause mortality with the use of primary ICD prophylaxis (HR 0.69, $95 \%$ CI 0.51-0.93). Finally, the SCD in the Heart Failure Trial (SCD-HeFT) compared standard medical therapy plus placebo versus standard medical therapy plus amiodarone versus ICD therapy [10•]. SCD-HeFT differed from the MADIT trials in allowing non-ischemic cardiomyopathy as an inclusion criterion, although systolic dysfunction (LVEF $\leq 35 \%$ ) and symptomatic heart failure (NYHA classes II or III) were still required for enrollment. The addition of amiodarone to standard medical therapy did not provide a mortality benefit (HR 1.06 vs. placebo, $97.5 \% \mathrm{CI}$ 0.86-1.30). Survival was enhanced in the ICD arm (HR 0.77 vs. placebo, $97.5 \%$ CI 0.62-0.96), independent of whether cardiomyopathy was ischemic or non-ischemic. Therefore, patients with acute heart failure should be included in outpatient referral for consideration of prophylactic defibrillator placement after stabilization and treatment.

In addition to defibrillation, implantable cardiac devices may be programmed to manage the beat-to-beat conduction of the failing heart. Prolonged ventricular contraction can exacerbate pre-existing cardiomyopathy, resulting in worsening contractile function as well as leading to unfavorable remodeling. The utilization of cardiac resynchronization therapy (CRT) with biventricular pacing is designed to overcome mechanical dyssynchrony using controlled synchronous depolarization of both ventricles. This technology has been demonstrated to enhance quality of life, decrease symptoms, and reverse remodeling [11]. The Multicenter InSync Randomized Clinical Evaluation (MIRACLE) trial enrolled 453 subjects with symptomatic heart failure (NYHA classes III or IV) characterized by ventricular dyssynchrony (QRS $\geq 130 \mathrm{~ms}$ ) and impaired systolic function (LVEF $\leq 35 \%)$ [12]. All subjects received an implantable cardiac device with CRT capacity, and were randomized to either 6 months of CRT or no pacing. At 6 months, the CRT group had demonstrated significant improvement in NYHA class, 6-min walk test, and quality of life. In addition, LVEF improved by a median of $5 \%$ and end diastolic volume decreased in the treatment group. Fewer hospitalizations for heart failure were required in the CRT group as well (83 vs. 363 hospital days). MIRACLE was not powered for mortality as an endpoint, which was statistically similar between groups at 6 months $(\mathrm{HR}=0.73$ favoring less mortality with CRT, $95 \%$ CI 0.34-1.54).

The Comparison of Medical Therapy, Pacing, and Defibrillator Therapy in Heart Failure trial randomized 1,520 patients with NYHA class III or IV heart failure, impaired systolic function (LVEF $\leq 35 \%$ ), and dysfunctional electrical conduction (QRS $\geq 120 \mathrm{~ms}$ and $\mathrm{PR}$ interval $\geq 150 \mathrm{~ms}$ ) to CRT with defibrillator (CRT-D), CRT alone, or optimal medical therapy [13•]. CRT and CRT-D therapy were associated with a substantially decreased rate of the primary endpoint of death or hospitalization (HR 0.8, $95 \%$ CI 0.68-0.95). The CRT-D group had a definite reduction in allcause mortality when compared to the optimal medical therapy group (HR 0.64, $95 \%$ CI 0.48-0.86).

To date, CRT has shown little benefit in patients with a narrow QRS complex [14]. It has been suggested that patients with echocardiographic evidence of dyssynchrony may benefit from resynchronization therapy despite a narrow QRS complex [15]; unfortunately this contention has not been prospectively borne out $[14,16]$. A large $(>1,250$ patient) ongoing trial, the EchoCRT study, is evaluating the effects of CRT on outcomes in narrow QRS patients with mechanical dyssynchrony. Conversely, prolonged QRS 
duration is not a marker for guaranteed improvement, as up to $30 \%$ of patients will not benefit despite CRT [17]. Regardless, multiple trials have demonstrated improvement in quality of life measures as well as survival in patients with severe heart failure, decreased ejection fraction, and electrical evidence of conduction disturbances [12, 13•, 18-22].

It is not our purpose to suggest that the recognition of implantable device indications and specialist referrals for such is the standard of care for the acute care setting. However, the penetration of these devices in the evidencebased, guideline recommended population (i.e., those with a class IA indication) is only about 40-50\% [23•]. Especially in underserved populations, the medical safety net provided by the ED/acute care setting may represent the best opportunity for referral for device therapies. Even in tertiary centers, standard referral patterns result in missed opportunities to get device-based therapies to at-risk patients [24]; physicians managing heart failure patients in the acute setting should be mindful of opportunities and resources that may decrease hospital admission recidivism and improve quality of life.

\section{Diagnostic Functions}

In order for implantable devices to perform the active functions of defibrillation, cardioversion, or pacing, they must record and interpret the patient's intrinsic cardiac rhythm data. Different devices store modestly different parameters, although there are some consistent metrics between devices and manufacturers. In addition to devices that record rate, rhythm, and response data, there are an increasing number of devices that collect advanced telemetry data, including physiological information such as heart rate variability, intrathoracic impedance, and patient activity level. Data from both basic and advanced monitoring parameters may be useful during the evaluation of the patient with suspected acute heart failure.

\section{Rhythm Data}

Atrial fibrillation is the most common dysrhythmia in patients with chronic heart failure; even patients thought to be maintaining sinus rhythm can experience clinically silent paroxysmal atrial fibrillation episodes [25]. New onset atrial fibrillation may be a worse marker for long-term survival, and many heart failure patients experience worsening symptoms with atrial fibrillation [26]. Conversely, there is also evidence that prolonged volume overload can also result in atrial tachydysrhythmias, perhaps as a result of electrical irritability due to atrial distension [27]. Discovery of atrial fibrillation as a precipitating event could lead to several different management options that would not have been apparent choices otherwise, such as initiating rate or rhythm controlling pharmacologic agents, instituting anticoagulation for stroke prophylaxis, or changing pacemaker programming parameters. In addition to atrial dysrhythmias, VT may also occur without overt clinical symptoms in the setting of chronic heart failure. CRT-based monitoring has shown both malignant ventricular arrhythmias as well as non-sustained VT to be associated with heart failure decompensation, similar to atrial tachydysrhythmias [28-30]. The presence of a high rate of ventricular dysrhythmia in the setting of decompensated heart failure should, of course, prompt optimization of electrolyte abnormalities, as well as consideration for exacerbations of ischemic disease as a potential etiology for the heart failure event. Finally, should the patient have a device not equipped with defibrillation capability, the presence of frequent ventricular tachydysrhythmias should also suggest the need for prompt consultation with the patient's electrophysiologist to consider defibrillator management.

\section{Heart Rate Variability}

There is an intrinsic variability in the heart rate of healthy individuals that reflects compensation for changes in physiologic demand as well as other diurnal patterns. As physiologic stress increases, this variance decreases due to a rise in sympathetic tone and an attenuation of the parasympathetic nervous system [31]. Implantable cardiac devices that monitor atrial depolarization can record atrial rates and calculate the variability in the intrinsic sinoatrial node function. The association between heart rate variability as a proxy measure for improved heart failure mechanics was established in a secondary analysis of the data from the CRTbased MIRACLE trial [12]. Those patients randomized to active CRT functionality experienced a substantial improvement in heart rate variability that was associated with improvement in cardiac function [32].

Heart rate variability was then linked as an independent predictor of outcomes, as opposed to a marker of response to therapy, in a prospective observational cohort study of 288 patients receiving a CRT device for NYHA class III or IV heart failure coupled with systolic dysfunction (LVEF $\leq 35 \%$ ) [33]. Over the course of a year, heart rate variability was significantly lower in patients experiencing hospitalization or death as opposed to those subjects who did not decompensate or had a mild decompensation not requiring hospitalization $(74 \pm 22$ vs. $90 \pm 22 \mathrm{~ms}$, $p<0.0001)$. The decrease in heart rate variability was notable at a median 16 days prior to hospitalization.

Unfortunately, a decrease in heart rate variability is not specific to acutely decompensated heart failure. Other 
illnesses and comorbidities that manifest with a ramping up of sympathetic tone also present with a decrease in heart rate variability, such as seen in exacerbation of chronic obstructive pulmonary disease [34] or various infectious states [35]. Examination of all parameters captured by the implantable device will give a more complete understanding of the patient's clinical picture.

\section{Patient Activity}

Accelerometers within the implanted device can provide a measurement of hours per day that the patient is moving and presumably physically active, although the actual degree of exertion is not captured within this measurement. As patients become more and more symptomatic with heart failure, exercise intolerance worsens and physical activity decreases [33]. Conversely, a study of patients receiving CRT pacing demonstrated an increase in daily activity levels that corresponded to improvements in NYHA class and exercise tolerance $(108 \pm 81 \mathrm{~min} /$ day at baseline vs. $225 \pm 140 \mathrm{~min} /$ day at 12 weeks, $p<0.001$ ) [36]. Patient activity levels have been shown to be less sensitive than decreased heart rate variability in predicting decompensation in the outpatient setting [33], although decreased physical activity levels have been shown to be predictive of subsequent heart failure decompensation within 30 days, when monitored in concert with other implantable device monitoring parameters (HR 5.5, $95 \%$ CI 3.4-8.8) [37].

\section{Intrathoracic Impedance}

The measurement of intrathoracic impedance utilizes changes in electrical conduction within the cardiopulmonary structures of the chest to gauge fluid overload. As the total amount of tissue fluid increases, resistance (also known as impedance) to conduction of an electrical impulse between a pulse generator and a sensor decreases. Therefore, a low impedance reading is a marker of pulmonic fluid congestion. Intrathoracic impedance determination is made using the pacemaker lead as the pulse generator and the device canister as the receptor. At the time of this writing, the only FDA-approved intrathoracic impedance technology for clinical use is proprietary to Medtronic, although other investigations are in progress.

Intrathoracic impedance has been evaluated as a predictor of heart failure decompensation in the outpatient arena in a number of studies. The proof of concept was established by $\mathrm{Yu}$ et al. [38], who demonstrated that impedance dropped an average of 18 days prior to hospitalization for fluid overload, and 15 days prior to the onset of worsening symptoms. Impedance values were also inversely correlated with pulmonary capillary wedge pressures obtained upon hospitalization. However, an elevated fluid index was not a perfect predictor of outcomesa threshold index of $60 \mathrm{ohm}$ days generated a sensitivity for hospitalization of $77 \%$ with a false positive alert rate of 1.5 alerts without subsequent hospitalization per patient year of observation. Similar performance has been noted in subsequent studies [28, 37, 39, 40].

Of potentially more impact within the acute care setting, Small et al. [41] have demonstrated, in a retrospective analysis of registry data derived from patients with CRTbased intrathoracic impedance monitoring, a low likelihood of hospitalization due to acute heart failure in subjects whose fluid index did not cross the set threshold $(0.14$ hospitalizations/patient years vs. 0.76 hospitalizations/ patient years in those patients with multiple threshold crossing events). It may be that an absence of threshold crossing, or at least a deterioration of the impedance values, may suggest that a dyspneic patient being evaluated in the acute setting has an etiology other than decompensated heart failure underlying their symptoms.

\section{Pressure Monitoring}

Several implantable devices that directly monitor intracardiac pressures are, at the time of this writing, in investigational status. The majority of the current generation of implantable hemodynamic monitors are purely data collection devices, as opposed to acting in combination with a pacemaker or defibrillator, although some have been combined with CRT and/or ICD devices. The HeartPOD system (St. Jude Medical, Minneapolis, MN) utilizes a wired pressure transducer in the left atrium to record cardiac data [42]. The HOMEOSTASIS (Hemodynamically Guided Home Self-Therapy in Severe Heart Failure Patients) trial evaluated the feasibility of providing this data directly to the patient by way of a handheld patient advisory module, which would collect the data from the implant and recommend changes in medication therapy (diuretics or vasodilators) based on algorithms pre-programmed by the physician [43]. The lack of a control group limits the conclusions that can be drawn from this small study $(n=40)$; however, given that the programmed algorithms advised medication changes on a frequent basis (53\% of days measured), this study provides a strong impetus for moving forward with a controlled trial of patient-facilitated management. That study, the LAPTOP$\mathrm{HF}$ trial, is now ongoing. In addition to the standalone HeartPOD system, LAPTOP-HF introduces a combined CRT-ICD-LAP monitoring system into clinical study. Additional devices under investigation include the CardioMEMS Heart Failure Sensor (CardioMEMS, Atlanta, 
GA), which uses a pressure transducer implanted in the pulmonary artery with wireless transmission of data to a handheld recorder [44]. In a 550-patient randomized controlled trial, the use of this wireless implantable hemodynamic monitoring system reduced the 6-month rate of heart failure hospitalization by $30 \%$ and, over prolonged followup averaging 15 months (range 1 day to 30 months), reduced heart failure hospitalizations by $39 \%$ [45]. Similarly, the RemonCHF device (Boston Scientific, Natick, MA) measures pulmonary artery pressures by way of a pressure transducer located in the pulmonary artery that provides on-demand interrogation powered by way of ultrasound transmission to and from a hand held unit that can be operated by the patient [46]. These devices are all still in trial stages, but offer exciting potential for patient management.

Finally, the first generation monitoring system, the Chronicle IHM (Medtronic Inc., Minneapolis, MN), is no longer in active investigation after having been rejected for clinical use by the FDA in 2007. The COMPASS-HF study demonstrated a non-significant reduction in heart failure events (hospitalization or ED visits requiring intravenous therapy) in patients receiving device-guided therapy compared to controls [47]. Although the intervention arm experienced fewer events ( 84 events in 44 patients vs. 113 events in 60 patients in the control arm) over 6 months, this difference was not statistically significant $(p=0.33)$.

\section{The Acute Care Setting}

To date, clinical trials of implantable device data have been directed at utilizing these parameters to keep patients from decompensating to the point of requiring ED- or hospitalbased care in the first place. In addition, the interrogation of these devices has generally fallen under the purview of the implanting physician. As a result, there is very little literature available that examines the actual use of device data in the diagnosis and management of decompensated heart failure once the patient has presented to the ED. In terms of preventing ED visits or hospitalization, clinical interventions based on remote monitoring of implantable device data has not yet lived up to expectations, with trials demonstrating neutral results in terms of rates of hospital care $[47,48]$. This is frustrating in that it is clearly demonstrable that abnormalities in cardiac parameters are associated with impending heart failure decompensation. Such diverse factors as heart rate variability [33], intrathoracic impedance $[38,39]$, ambulatory right ventricular pressures [39, 49, 50], and increased atrial tachydysrhythmia burden [27], alone or in various combination [37], are all associated with an increased risk of decompensation, and all can be recorded and transmitted to a clinician remotely.
Once the patient with an implantable cardiac device presents acutely with symptoms such as dyspnea that may be due to heart failure decompensation, several challenges present themselves to the treating physician. First, the doctor must determine if the patient's symptoms are truly due to decompensated heart failure. Given that the patient has severe enough heart failure to warrant placement of an implantable device, one might consider the a priori probability of decompensation to be relatively high. However, the use of implantable device data may either serve as valuable confirmation of the presence of acute heart failure, or suggest another pathologic process is the etiology of the patient's symptoms. However, at this time no studies have evaluated the diagnostic performance of implantable cardiac device data in differentiating acutely decompensated heart failure from other disease entities that may present in a similar fashion.

Once the physician has determined that acute heart failure is present, the next step should be to determine how best to treat the patient. The modalities chosen (diuresis, afterload reduction, inotropic support, and airway intervention) will depend greatly on the perfusion status and the volume status of the patient, as well as the clinical severity of the presentation. Although respiratory compromise and systemic perfusion will be fairly obvious with routine exam, volume status may at times be difficult to discernespecially in the obese. Devices that measure volumetric data, such as intrathoracic impedance or direct atrial pressure monitors, may provide insight into the degree of appropriate diuresis required. This may allow the physician to adequately remove volume while avoiding the complications of overdiuresis and subsequent renal stress.

Finally, in the patient with acute heart failure, it becomes critical to understand why the patient decompensated in the first place. Examination of the longitudinal data contained within the implantable device may provide key insights as to the underlying mechanisms that brought the patient to this state. Rhythm data may indicate increasing frequency of atrial fibrillation, which could require pacemaker reprogramming, pharmacologic management, or even A-V nodal ablation to improve hemodynamic function. Given that abnormalities in heart rate variability, patient activity levels, and fluid accumulation precede clinical decompensation by several days [29, 33, 38, 39], going over temporal data with the patient to evaluate medication, diet, and other lifestyle events such as exacerbations of comorbid illnesses may establish a causative link to behaviors or illnesses that led to the acute decompensation.

Unfortunately, these possibilities, although grounded in a solid conceptual framework, have yet to be validated beyond anecdotal experience. As stated previously, the research effort to date has been directed at keeping the patient from requiring acute care in the first place. While 
this is definitely a worthy goal and would benefit the patient, the truth of the matter remains that over one million hospitalizations for heart failure will occur annually [51・•]. There definitely remains a need for research to establish the additive value of basic and advanced implantable device data for the evaluation and management of the patient with suspected acute heart failure. Until such research is established, however, it is certainly reasonable for those of us caring for patients who have this data readily available to evaluate and consider the recorded information in the context of the patient's presentation.

\section{Conclusion}

The ability of non-implanting physicians to view this device data is becoming increasingly available in the acute care setting. Given the potential for these devices to assist in the acute diagnosis of worsening heart failure and to guide initial therapies, knowledge of the strengths and weaknesses of device-based diagnostics is becoming a must for ED- and hospital-based personnel.

Disclosures B. Hiestand is a consultant for NewMentor, Inc., Insight PD, Grants from Medtronics Inc., Biosite Inc., Heartscape International, Nanosphere Inc., Mitsubishi Medience, The Medicines Company, Sanofi Aventis, Dyax. S. Laribi: nothing to disclose. A. Mebazaa: nothing to disclose.

\section{References}

Papers of particular interest, published recently, have been highlighted as:

- Of importance

•- Of major importance

1. • McMurray JJ, Adamopoulos S, Anker SD, et al. ESC Guidelines for the Diagnosis and Treatment of Acute and Chronic Heart Failure 2012: The Task Force for the Diagnosis and Treatment of Acute and Chronic Heart Failure 2012 of the European Society of Cardiology. Developed in collaboration with the Heart Failure Association (HFA) of the ESC. Eur Heart J. 2012;33:1787-847. These are the most recent guidelines published by the European Society of Cardiology for heart failure management.

2. Peacock WF. Emergency department observation unit management of heart failure. Crit Pathw Cardiol. 2003;2:207-20.

3. Peacock WF, Emerman C, Costanzo MR, et al. Early vasoactive drugs improve heart failure outcomes. Congest Heart Fail. 2009;15:256-64.

4. Zipes DP, Camm AJ, Borggrefe M, et al. ACC/AHA/ESC 2006 Guidelines for Management of Patients With Ventricular Arrhythmias and the Prevention of Sudden Cardiac Death: a report of the American College of Cardiology/American Heart Association Task Force and the European Society of Cardiology Committee for Practice Guidelines (writing committee to develop Guidelines for Management of Patients With Ventricular
Arrhythmias and the Prevention of Sudden Cardiac Death): developed in collaboration with the European Heart Rhythm Association and the Heart Rhythm Society. Circulation. 2006;114:e385-484.

5. Myerburg RJ. Sudden cardiac death: exploring the limits of our knowledge. J Cardiovasc Electrophysiol. 2001;12:369-81.

6. MERIT-HF Study Group. Effect of metoprolol CR/XL in chronic heart failure: Metoprolol CR/XL Randomised Intervention Trial in Congestive Heart Failure (MERIT-HF). Lancet. 1999;353:2001-7.

7. Epstein AE, DiMarco JP, Ellenbogen KA, et al. ACC/AHA/HRS 2008 Guidelines for Device-Based Therapy of Cardiac Rhythm Abnormalities: a report of the American College of Cardiology/ American Heart Association Task Force on Practice Guidelines (Writing Committee to Revise the ACC/AHA/NASPE 2002 Guideline Update for Implantation of Cardiac Pacemakers and Antiarrhythmia Devices): developed in collaboration with the American Association for Thoracic Surgery and Society of Thoracic Surgeons. Circulation. 2008;117:e350-408.

8. Moss AJ, Hall WJ, Cannom DS, et al. Improved survival with an implanted defibrillator in patients with coronary disease at high risk for ventricular arrhythmia. Multicenter Automatic Defibrillator Implantation Trial Investigators. N Engl J Med. 1996; 335:1933-40.

9. Moss AJ, Zareba W, Hall WJ, et al. Prophylactic implantation of a defibrillator in patients with myocardial infarction and reduced ejection fraction. N Engl J Med. 2002;346:877-83.

10. - Bardy GH, Lee KL, Mark DB, et al. Amiodarone or an implantable cardioverter-defibrillator for congestive heart failure. N Engl J Med. 2005;352:225-37. This article demonstrates the mortality benefit of ICD placement in $\mathrm{HF}$, regardless of ischemic vs. non-ischemic etiology.

11. Solomon SD, Foster E, Bourgoun M, et al. Effect of cardiac resynchronization therapy on reverse remodeling and relation to outcome: multicenter automatic defibrillator implantation trial: cardiac resynchronization therapy. Circulation. 2010;122:985-92.

12. Abraham WT, Fisher WG, Smith AL, et al. Cardiac resynchronization in chronic heart failure. N Engl J Med. 2002;346:1845-53.

13. - Bristow MR, Saxon LA, Boehmer J, et al. Cardiac-resynchronization therapy with or without an implantable defibrillator in advanced chronic heart failure. N Engl J Med. 2004;350: 2140-50. This article definitively advances the mortality benefit associated with CRT.

14. Beshai JF, Grimm RA, Nagueh SF, et al. Cardiac-resynchronization therapy in heart failure with narrow QRS complexes. N Engl J Med. 2007;357:2461-71.

15. van Bommel RJ, Gorcsan J III, Chung ES, et al. Effects of cardiac resynchronisation therapy in patients with heart failure having a narrow QRS Complex enrolled in PROSPECT. Heart. 2010;96: 1107-13.

16. Chung ES, Leon AR, Tavazzi L, et al. Results of the Predictors of Response to CRT (PROSPECT) trial. Circulation. 2008;117: 2608-16.

17. Bax JJ, Abraham T, Barold SS, et al. Cardiac resynchronization therapy: Part 1-issues before device implantation. J Am Coll Cardiol. 2005;46:2153-67.

18. Young JB, Abraham WT, Smith AL, et al. Combined cardiac resynchronization and implantable cardioversion defibrillation in advanced chronic heart failure: the MIRACLE ICD Trial. JAMA. 2003;289:2685-94.

19. Cleland JG, Daubert JC, Erdmann E, et al. The effect of cardiac resynchronization on morbidity and mortality in heart failure. N Engl J Med. 2005;352:1539-49.

20. Cleland JG, Daubert JC, Erdmann E, et al. Longer-term effects of cardiac resynchronization therapy on mortality in heart failure [the CArdiac REsynchronization-Heart Failure (CARE-HF) trial extension phase]. Eur Heart J. 2006;27:1928-32. 
21. Linde C, Abraham WT, Gold MR, et al. Randomized trial of cardiac resynchronization in mildly symptomatic heart failure patients and in asymptomatic patients with left ventricular dysfunction and previous heart failure symptoms. J Am Coll Cardiol. 2008;52:1834-43.

22. Moss AJ, Hall WJ, Cannom DS, et al. Cardiac-resynchronization therapy for the prevention of heart-failure events. N Engl J Med. 2009;361:1329-38.

23. - Fonarow GC, Yancy CW, Albert NM, et al. Heart failure care in the outpatient cardiology practice setting: findings from IMPROVE HF. Circ Heart Fail. 2008;1:98-106. This article strongly suggests that our current practices are not sufficient in delivering evidence based care with regards to implantable cardiac devices in heart failure.

24. Bradfield J, Warner A, Bersohn MM. Low referral rate for prophylactic implantation of cardioverter-defibrillators in a tertiary care medical center. Pacing Clin Electrophysiol. 2009;32(Suppl 1):S194-7.

25. Caldwell JC, Contractor H, Petkar S, et al. Atrial fibrillation is under-recognized in chronic heart failure: insights from a heart failure cohort treated with cardiac resynchronization therapy. Europace. 2009;11:1295-300.

26. Wang TJ, Larson MG, Levy D, et al. Temporal relations of atrial fibrillation and congestive heart failure and their joint influence on mortality: the Framingham Heart Study. Circulation. 2003;107:2920-5.

27. Jhanjee R, Templeton GA, Sattiraju S, et al. Relationship of paroxysmal atrial tachyarrhythmias to volume overload: assessment by implanted transpulmonary impedance monitoring. Circ Arrhythm Electrophysiol. 2009;2:488-94.

28. Perego GB, Landolina M, Vergara G, et al. Implantable CRT device diagnostics identify patients with increased risk for heart failure hospitalization. J Interv Card Electrophysiol. 2008;23: $235-42$.

29. Moore HJ, Peters MN, Franz MR, et al. Intrathoracic impedance preceding ventricular tachyarrhythmia episodes. Pacing Clin Electrophysiol. 2010;33:960-6.

30. Ip JE, Cheung JW, Park D, et al. Temporal associations between thoracic volume overload and malignant ventricular arrhythmias: a study of intrathoracic impedance. J Cardiovasc Electrophysiol. 2010;22:293-9.

31. Goldsmith RL, Bigger JT, Bloomfield DM, et al. Long-term carvedilol therapy increases parasympathetic nervous system activity in chronic congestive heart failure. Am J Cardiol. 1997;80:1101-4.

32. Adamson PB, Kleckner KJ, VanHout WL, et al. Cardiac resynchronization therapy improves heart rate variability in patients with symptomatic heart failure. Circulation. 2003;108:266-9.

33. Adamson PB, Smith AL, Abraham WT, et al. Continuous autonomic assessment in patients with symptomatic heart failure: prognostic value of heart rate variability measured by an implanted cardiac resynchronization device. Circulation. 2004;110:2389-94.

34. Camillo CA, Pitta F, Possani HV, et al. Heart rate variability and disease characteristics in patients with COPD. Lung. 2008;186: 393-401.

35. Ahmad S, Tejuja A, Newman KD, et al. Clinical review: a review and analysis of heart rate variability and the diagnosis and prognosis of infection. Crit Care. 2009;13:232.

36. Braunschweig F, Mortensen PT, Gras D, et al. Monitoring of physical activity and heart rate variability in patients with chronic heart failure using cardiac resynchronization devices. Am J Cardiol. 2005;95:1104-7.

37. Whellan DJ, Ousdigian KT, Al Khatib SM, et al. Combined heart failure device diagnostics identify patients at higher risk of subsequent heart failure hospitalizations: results from PARTNERS
HF (Program to Access and Review Trending Information and Evaluate Correlation to Symptoms in Patients With Heart Failure) study. J Am Coll Cardiol. 2010;55:1803-10.

38. Yu CM, Wang L, Chau E, et al. Intrathoracic impedance monitoring in patients with heart failure: correlation with fluid status and feasibility of early warning preceding hospitalization. Circulation. 2005;112:841-8.

39. Vanderheyden M, Houben R, Verstreken S, et al. Continuous monitoring of intrathoracic impedance and right ventricular pressures in patients with heart failure. Circ Heart Fail. 2010;3:370-7.

40. Catanzariti D, Lunati M, Landolina M, et al. Monitoring intrathoracic impedance with an implantable defibrillator reduces hospitalizations in patients with heart failure. Pacing Clin Electrophysiol. 2009;32:363-70.

41. Small RS, Wickemeyer W, Germany R, et al. Changes in intrathoracic impedance are associated with subsequent risk of hospitalizations for acute decompensated heart failure: clinical utility of implanted device monitoring without a patient alert. J Card Fail. 2009;15:475-81.

42. Ritzema J, Melton IC, Richards AM, et al. Direct left atrial pressure monitoring in ambulatory heart failure patients: initial experience with a new permanent implantable device. Circulation. 2007;116:2952-9.

43. Ritzema J, Troughton R, Melton I, et al. Physician-directed patient self-management of left atrial pressure in advanced chronic heart failure. Circulation. 2010;121:1086-95.

44. Verdejo HE, Castro PF, Concepcion R, et al. Comparison of a radiofrequency-based wireless pressure sensor to Swan-Ganz catheter and echocardiography for ambulatory assessment of pulmonary artery pressure in heart failure. J Am Coll Cardiol. 2007;50:2375-82.

45. Abraham WT, Adamson PB, Bourge RC, Holder P. Wireless pulmonary artery haemodynamic monitoring in chronic heart failure: a randomised controlled trial. Lancet. 2011;377:658-66.

46. Hoppe UC, Vanderheyden M, Sievert H, et al. Chronic monitoring of pulmonary artery pressure in patients with severe heart failure: multicentre experience of the monitoring Pulmonary Artery Pressure by Implantable device Responding to Ultrasonic Signal (PAPIRUS) II study. Heart. 2009;95:1091-7.

47. Bourge RC, Abraham WT, Adamson PB, et al. Randomized controlled trial of an implantable continuous hemodynamic monitor in patients with advanced heart failure: the COMPASSHF study. J Am Coll Cardiol. 2008;51:1073-9.

48. Zile MR, Bourge RC, Bennett TD, et al. Application of implantable hemodynamic monitoring in the management of patients with diastolic heart failure: a subgroup analysis of the COMPASS-HF trial. J Card Fail. 2008;14:816-23.

49. Stevenson LW, Zile M, Bennett TD, et al. Chronic ambulatory intracardiac pressures and future heart failure events. Circ Heart Fail. 2010;3:580-7.

50. Zile MR, Bennett TD, St John SM, et al. Transition from chronic compensated to acute decompensated heart failure: pathophysiological insights obtained from continuous monitoring of intracardiac pressures. Circulation. 2008;118:1433-41.

51. • Hunt SA, Abraham WT, Chin MH, et al. 2009 focused update incorporated into the ACC/AHA 2005 Guidelines for the Diagnosis and Management of Heart Failure in Adults: a report of the American College of Cardiology Foundation/American Heart Association Task Force on Practice Guidelines: developed in collaboration with the International Society for Heart and Lung Transplantation. Circulation. 2009;119:391-479. These are the most recent guidelines published by the American College of Cardiology/American Heart Association for heart failure management. 